\title{
Reciprocal Changes in Sagittal Alignment in Adolescent Idiopathic Scoliosis Patients Following Strategic Pedicle Screw Fixation
}

\author{
Srikanth Reddy Dumpa, Ajoy Prasad Shetty, Siddharth N. Aiyer, Rishi Mugesh Kanna, S Rajasekaran \\ Department of Spine Surgery, Ganga Hospital, Coimbatore, India
}

\section{Study Design: Retrospective observational study.}

Purpose: To analyze the effect of low-density (LD) strategic pedicle screw fixation on the correction of coronal and sagittal parameters in adolescent idiopathic scoliosis (AIS) patients.

Overview of Literature: LD screw fixation achieves favorable coronal correction, but its effect on sagittal parameters is not well established. AIS is often associated with decreased thoracic kyphosis (TK), and the use of multi-level pedicle screws may result in further flattening of the sagittal profile.

Methods: A retrospective analysis was performed on 92 patients with AIS to compare coronal and sagittal parameters preoperatively and at 2-year follow-up. All patients underwent posterior correction via LD strategic pedicle screw fixation. Radiographs were analyzed for primary Cobb angle (PCA), coronal imbalance, cervical sagittal angle (CSA), TK, lumbar lordosis (LL), pelvic incidence, pelvic tilt (PT), sacral slope (SS), C7 plumb line, spino-sacral angle, curve flexibility, and screw density.

Results: PCA changed significantly from $57.6^{\circ} \pm 13.9^{\circ}$ to $19^{\circ} \pm 8.4^{\circ}(p<0.0001)$ with $67 \%$ correction, where the mean curve flexibility was $41 \%$ and screw density was $68 \%$. Regional sagittal parameters did not change significantly, including CSA (from $10.76^{\circ}$ to $10.56^{\circ}, p=0.893$ ), TK (from $24.4^{\circ}$ to $22.8^{\circ}, p=0.145$ ), and LL (from $50.3^{\circ}$ to $51.1^{\circ}, p=0.415$ ). However, subgroup analysis of the hypokyphosis group $\left(<10^{\circ}\right)$ and the hyperkyphosis group $\left(>40^{\circ}\right)$ showed significant correction of TK $(p<0.0001$ in both). Sacro-pelvic parameters showed a significant decrease of PT and increase of SS, suggesting a reduction in pelvic retroversion SS (from $37^{\circ}$ to $40^{\circ}$, $p=0.0001$ ) and PT (from $15^{\circ}$ to $14^{\circ}, p=0.025$ ).

Conclusions: LD strategic pedicle screw fixation provides favorable coronal correction and improves overall sagittal sacro-pelvic parameters. This technique does not cause significant flattening of TK and results in a favorable restoration of TK in patients with hypokyphosis or hyperkyphosis.

Keywords: Scoliosis; Spinal fusion; Pedicle screws; Low density; Radiological outcome

This is the 2017 ASSI-Asian Spine Journal Best Paper Award.

Received Jan 23, 2018; Accepted Feb 3, 2018

Corresponding author: Ajoy Prasad Shetty

Department of Spine Surgery, Ganga Hospital, 313, Mettupalayam road, Coimbatore, India

Tel: +91-9344833797, Fax: +91-422-4383863, E-mail: ajoyshetty@gmail.com 


\section{Introduction}

The primary goals of surgical treatment of adolescent idiopathic scoliosis (AIS) are preventing further curve progression; attaining a balanced spine; and correcting deformities in coronal, sagittal, and transverse planes [1]. Use of segmental pedicle screws for deformity correction was first demonstrated by Suk et al. [2] and several studies have since reported safety and efficacy of pedicle screws in scoliosis deformity correction [3]. However, because of its stiffness, controversy exists over the ability of all-screw instrumentation to restore thoracic kyphosis (TK) $[4,5]$. Low-density (LD) pedicle screw constructs utilize fewer pedicle screws that are strategically positioned, thereby reducing neurological risk, surgical time, blood loss, and surgical costs [6]. Studies on the use of different screw density constructs in AIS by Davis and Dunn [7], Kemppainen et al. [8], and Larson et al. [9] have demonstrated satisfactory results with LD screw constructs in comparison with high-density (HD) screw constructs. Though LD screw constructs achieve favorable results, their effect on sagittal parameters is not well established. Therefore, we aimed to study the effect of LD screw fixation in AIS by quantifying changes in sagittal spinal parameters in addition to those in coronal parameters.

\section{Materials and Methods}

\section{Study design and inclusion criteria}

Ninety two patients treated with surgical correction for AIS during the period from January 2012 to December 2014 were included in this retrospective, single center study. The study was approved by the institutional review board and was conducted in accordance with the ethical standards of the declaration of Helsinki.

Inclusion criteria were as follows: AIS with Lenke curve type I-VI, surgical treatment with posterior instrumented fusion with pedicle screws and rod construct, absence of neurological deficit, a normal preoperative magnetic resonance imaging (MRI) of the spinal axis, a and minimum of 2 years of follow-up. Exclusion criteria were as follows: neuromuscular or congenital scoliosis, presence of abnormal MRI, and patients who had undergone anterior surgery.

\section{Data collection and radiographic analysis}

Demographic data included age, sex, curve patterns, Rissers stage, type of surgery performed, number of fusion segments, and screw density.

Radiographic data were analyzed on anteroposterior and lateral radiographic views of the entire spine in the upright position. Curve flexibility was assessed on supine side-bending films. Preoperative and postoperative data were analyzed with a minimum 2-year follow-up. We analyzed the following parameters: primary Cobb angle (PCA), coronal imbalance (CI), cervical sagittal angle (CSA), TK, lumbar lordosis (LL), pelvic incidence (PI), pelvic tilt (PT), sacral slope (SS), C7 plumb line (C7PL), spino-sacral angle (SSA), and screw density. All radiological parameters were assessed as per standard norms described in the literature (Fig. 1A-C). If the plumb line fell to the left of the central sacral vertical line (CSVL), $\mathrm{CI}$ was considered negative and if the plumb line fell to the right, $\mathrm{CI}$ was considered positive. If the plumb line fell behind the posterosuperior corner of the first sacral vertebra, C7PL was considered negative and if the plumb line fell ahead, C7PL was considered positive. Screw density was calculated using the following formula: screw density=number of pedicle screws placed/number of pedicles in fusion segments $\times 100$.

\section{Surgical technique}

All surgeries were performed under general anesthesia with the patient in prone position and under neuromonitoring surveillance. Pedicle screws were inserted by means of a "free-hand" technique. Strategic pedicle screw fixation (SPSF) was performed in the following manner. The cranial and caudal foundation typically consisted of four screws. Pedicle screws were then strategically placed at the intervening levels, typically including more screws in the concavity of the deformity (Fig. 1D). To improve curve flexibility, facetectomies and Ponte osteotomies were included in individual cases. Next, manually pre-contoured 5.5-mm titanium rods were inserted, and reduction of the deformity was accomplished through a combination of rod derotation, translational reduction, and in situ correction, if required. Posterior element decortication and bone grafting were then performed via autograft and allograft. 


\section{Statistics}

Radiographic data was stored and analyzed using picture archiving and communication system. Data analysis was done using the software IBM SPSS ver. 22.0 (IBM Corp., Armonk, NY, USA) in pre- and postoperative cohorts of all patients. Based on the extent of TK, subgroup analysis was performed in the hypokyphosis $\left(<10^{\circ}\right)$, normokyphosis $\left(10^{\circ}-40^{\circ}\right)$, and hyperkyphosis $\left(>40^{\circ}\right)$ groups. Student t-test was used for assessing statistical significance of differences between variables. In all comparisons performed, the statistical significance level was set at $p<0.05$.

\section{Results}

\section{Demographic results}

The study included 92 AIS patients (82 females and 10

Table 1. Radiographic results of the entire cohort ( $n=92$ )

\begin{tabular}{|c|c|c|c|}
\hline Parameter & Range & Mean \pm standard deviation & $p$-value \\
\hline Primary Cobb angle $\left({ }^{\circ}\right)$ & & & $<0.0001$ \\
\hline Preop & 35 to 97 & $57.7 \pm 13.9$ & \\
\hline Postop ${ }^{\text {a) }}$ & 2 to 50 & $19.02 \pm 8.4$ & \\
\hline Coronal imbalance (mm) & & & 0.157 \\
\hline Preop & -56 to 48 & $-7.2 \pm 24.05$ & \\
\hline Postop & -41 to 25 & $-3.4 \pm 14.9$ & \\
\hline C2-C6 angle (cervical sagittal angle) $\left(^{\circ}\right)$ & & & 0.893 \\
\hline Preop & -18 to 48 & $10.76 \pm 15.4$ & \\
\hline Postop & -9 to 41 & $10.56 \pm 11.9$ & \\
\hline Thoracic kyphosis $\left({ }^{\circ}\right)$ & & & 0.145 \\
\hline Preop & 3 to 56 & $24.46 \pm 11.7$ & \\
\hline Postop & 7 to 48 & $22.79 \pm 8.4$ & \\
\hline Lumbar lordosis $\left({ }^{\circ}\right)$ & & & 0.419 \\
\hline Preop & 31 to 78 & $50.3 \pm 10.6$ & \\
\hline Postop & 29 to 80 & $51.1 \pm 10.3$ & \\
\hline Pelvic incidence $\left({ }^{\circ}\right)$ & & & 0.012 \\
\hline Preop & 33 to 71 & $52 \pm 10.1$ & \\
\hline Postop & 22 to 71 & $54 \pm 10.2$ & \\
\hline Sacral slope $\left({ }^{\circ}\right)$ & & & $<0.001$ \\
\hline Preop & 17 to 65 & $37 \pm 8.2$ & \\
\hline Postop & 19 to 61 & $40 \pm 8.1$ & \\
\hline Pelvic tilt $\left({ }^{\circ}\right)$ & & & 0.025 \\
\hline Preop & 1 to 47 & $15 \pm 8.1$ & \\
\hline Postop & -10 to 41 & $14 \pm 8.3$ & \\
\hline Sagittal vertical axis (C7 plumb line) (mm) & & & $<0.0001$ \\
\hline Preop & -92 to 76 & $-8.2 \pm 36.8$ & \\
\hline Postop & -62 to 104 & $10.6 \pm 30.3$ & \\
\hline Spino-sacral angle $\left({ }^{\circ}\right)$ & & & 0.418 \\
\hline Preop & 109 to 150 & $127.5 \pm 8.5$ & \\
\hline Postop & 107.4 to 146.7 & $126.8 \pm 7.0$ & \\
\hline
\end{tabular}

Preop, preoperative; Postop, postoperative.

${ }^{\text {a) }}$ All postoperative values mentioned are values measured at final follow-up (all patients had a minimum of a 2-year follow-up). 
males), with an average age of 15.7 years. All the patients had a fused triradiate cartilage, and, based on the Lenke classification, there were 40 type I, 3 type II, 13 type III, 1 type IV, 25 type V, and 10 type VI patients.

\section{Radiographic results}

Preoperative analysis of the cohort demonstrated an average PCA of $57.7^{\circ} \pm 13.9^{\circ}$ (range, $35^{\circ}$ to $97^{\circ}$ ), which decreased to $19.0^{\circ} \pm 8.4^{\circ}$ (range, $2^{\circ}$ to $50^{\circ}$ ) at final followup, with a significant difference $(p<0.0001)$ (Table 1$)$. Mean primary curve flexibility was $41 \%$, screw density was $68 \%$, and the correction achieved in PCA was $67 \%$. Preoperative CI of $-7.2 \pm 24.05 \mathrm{~mm}$ (range, -56 to $48 \mathrm{~mm}$ ) improved to $-3.4 \pm 14.9 \mathrm{~mm}$ (range, -41 to $25 \mathrm{~mm}$ ) postoperatively; however, the change was not statistically significant $(p=0.157)$. With respect to regional sagittal parameters, no significant difference was found in CSA, TK, or LL. Mean preoperative and postoperative CSA were $10.76^{\circ} \pm 15.4^{\circ}$ and $10.56^{\circ} \pm 11.9^{\circ}$, respectively. Mean preoperative and postoperative TK were $24.46^{\circ} \pm 11.7^{\circ}$ and $22.79^{\circ} \pm 8.4^{\circ}$, respectively. Mean preoperative and postoperative LL were $50.3^{\circ} \pm 10.6^{\circ}$ and $51.1^{\circ} \pm 10.3^{\circ}$, respectively. Sacro-pelvic parameters (PI, SS, and PT) showed statistically significant changes in postoperative radiographs. Preoperatively, PI, SS, and PT were $52^{\circ} \pm 10.1^{\circ}, 37^{\circ} \pm 8.2^{\circ}$, and $15^{\circ} \pm 8.1^{\circ}$, respectively, changing to $54^{\circ} \pm 10.2^{\circ}$, $40^{\circ} \pm 8.1^{\circ}$, and $14^{\circ} \pm 8.3^{\circ}$, respectively, at final follow-up. The global sagittal parameter C7PL showed an $18.8 \mathrm{~mm}$ anterior shift with a preoperative value of $-8.2 \pm 36.8 \mathrm{~mm}$ and a postoperative value of $10.6 \pm 30.3 \mathrm{~mm}$ with a significant difference $(p<0.0001)$. SSA showed a decrease of $0.7^{\circ}$ without a significant difference $(p=0.418)$.

\section{Analysis based on thoracic kyphosis}

Based on the thoracic sagittal modifier, 11 patients showed preoperative thoracic hypokyphosis, 73 showed normokyphosis, and eight showed hyperkyphosis. In all the subgroups, coronal parameters were significantly corrected. In the hypokyphosis and hyperkyphosis groups, TK values were $7.0^{\circ}$ and $48.7^{\circ}$, respectively. These changed postoperatively to $16.7^{\circ}$ and $25.7^{\circ}$, respectively, and the changes were statistically significant $(p<0.0001)$ in both the subgroups. In the normokyphosis subgroup, there was a decrease in TK from $24.4^{\circ}$ to $23.3^{\circ}$; however, this change was not statistically significant (Table 2 ).

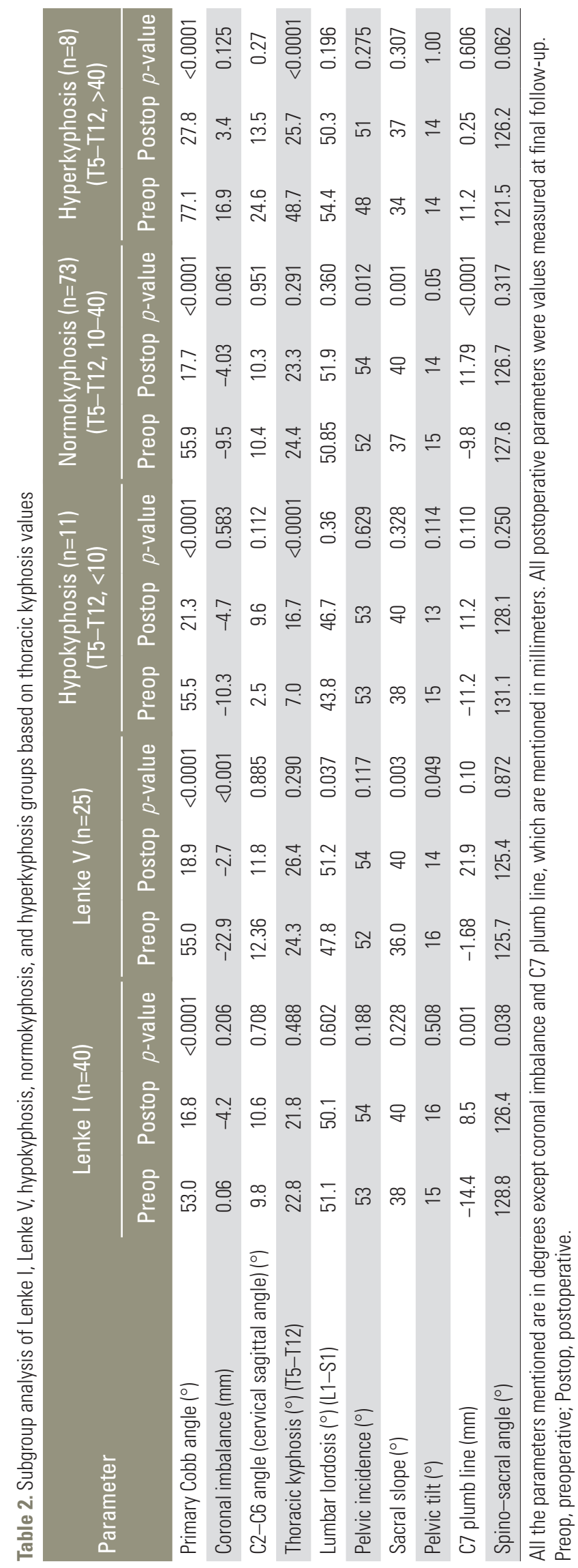



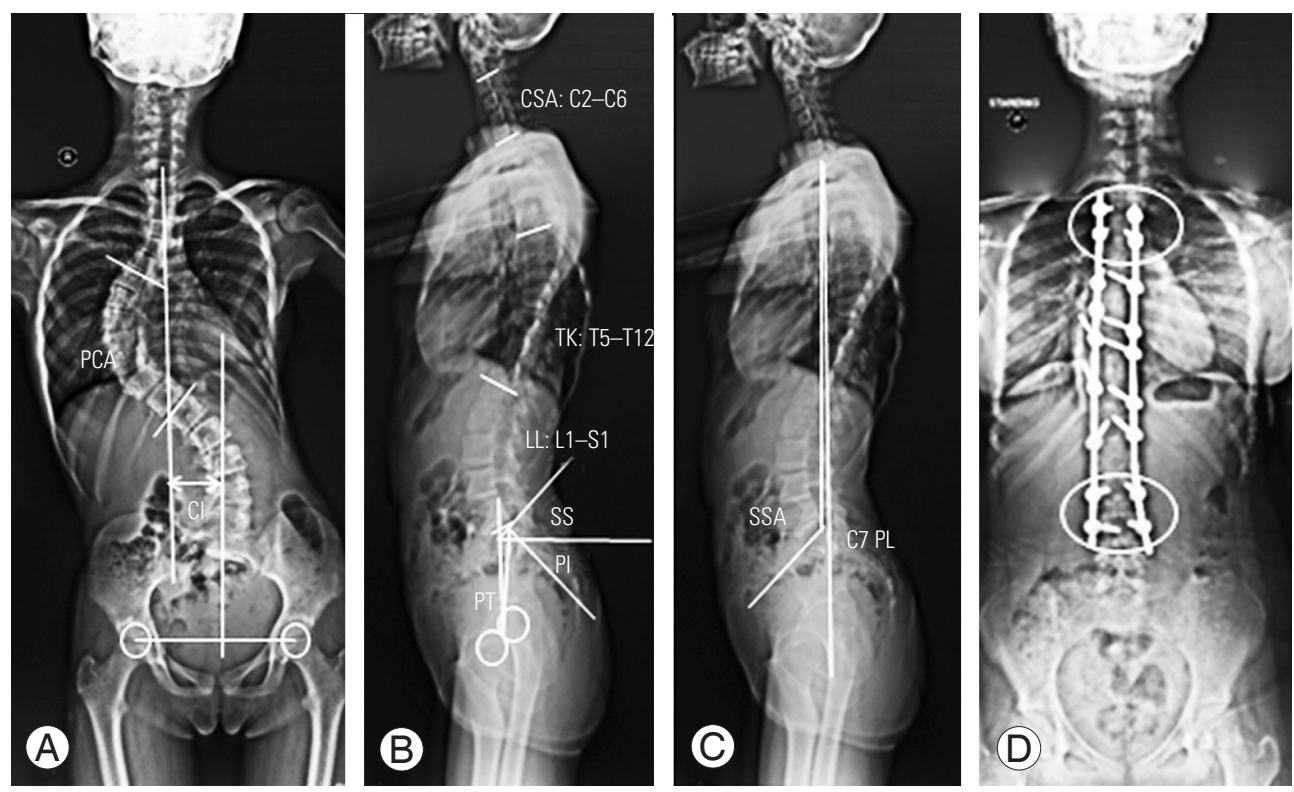

Fig. 1. Measurement of various radiographic parameters including both coronal (A) and sagittal parameters (B, C). Postoperative radiograph showing strategic pedicle screw fixation for the cranial and caudal foundation along with intermittent strategic placement of screws (D). PCA, primary Cobb angle; $\mathrm{Cl}$, coronal imbalance; CSA, cervical sagittal angle; TK, thoracic kyphosis; LL, lumbar lordosis; SS, sacral slope; PI, pelvic incidence; PT, pelvic tilt; SSA, spino-sacral angle; C7PL, C7 plumb line.

\section{Analysis in the Lenke I and Lenke V subgroups}

Statistical significant differences in PCA correction were observed in all the Lenke subgroups $(p<0.0001)$. In the Lenke I group, global spinal parameters (C7PL and SSA) were significantly altered compared with pre and postoperative data, and the remaining parameters did not show any significant differences. In the Lenke V subgroup, CI, LL, SS, and PT showed statistically significant differences between pre and postoperative cohorts (Table 2).

\section{Discussion}

The purpose of this study was to demonstrate the effect of LD SPSF on sagittal parameters in AIS. AIS has been associated with decrease in TK [10]. Restoration of TK in AIS is important to prevent increased risk of adjacent-segment disease, disc degeneration, positive sagittal balance, and back pain [11]. Studies on LD screw constructs by Davis and Dunn [7], Bharucha et al. [12], and Shen et al. [13] have noted decreases in TK, and, on the contrary, Wang et al. [14] noted an increase in TK. Therefore, restoration of TK due to LD screw construct has been variable in reported studies, and these studies were restricted mainly to TK and LL. Further, they did not consider reciprocal changes occurring in other sagittal spinal parameters. In the present study, we analyzed not only corrections in coronal parameters but also those in sagittal parameters and compared the parameters with those in the normal adolescent population.

Low implant density is poorly defined in the literature and different techniques, such as the interval pedicle screw strategy, skipped pedicle screw strategy, and key vertebral pedicle screw strategy, have been described [14]. We use strategic pedicle screw placement in which the cranial and caudal foundation typically comprised four screws, and intervening pedicle screws were placed strategically, typically including more screws in the concavity of the deformity (Fig. 2).

\section{Coronal parameters}

Davis and Dunn [7] reported that strategic screw placement achieved $65.1 \%$ improvement in the Cobb angle compared with $69.5 \%-79.6 \%$ in studies with higher screw density constructs $[7,15]$. They attributed lower coronal plane correction to lesser curve flexibility and to lesser metal density of $52.2 \%$. Li et al. [16] obtained an average of $74 \%$ correction with a mean preoperative Cobb angle of 61.87 in Lenke I curves with LD screw constructs. Bha- 

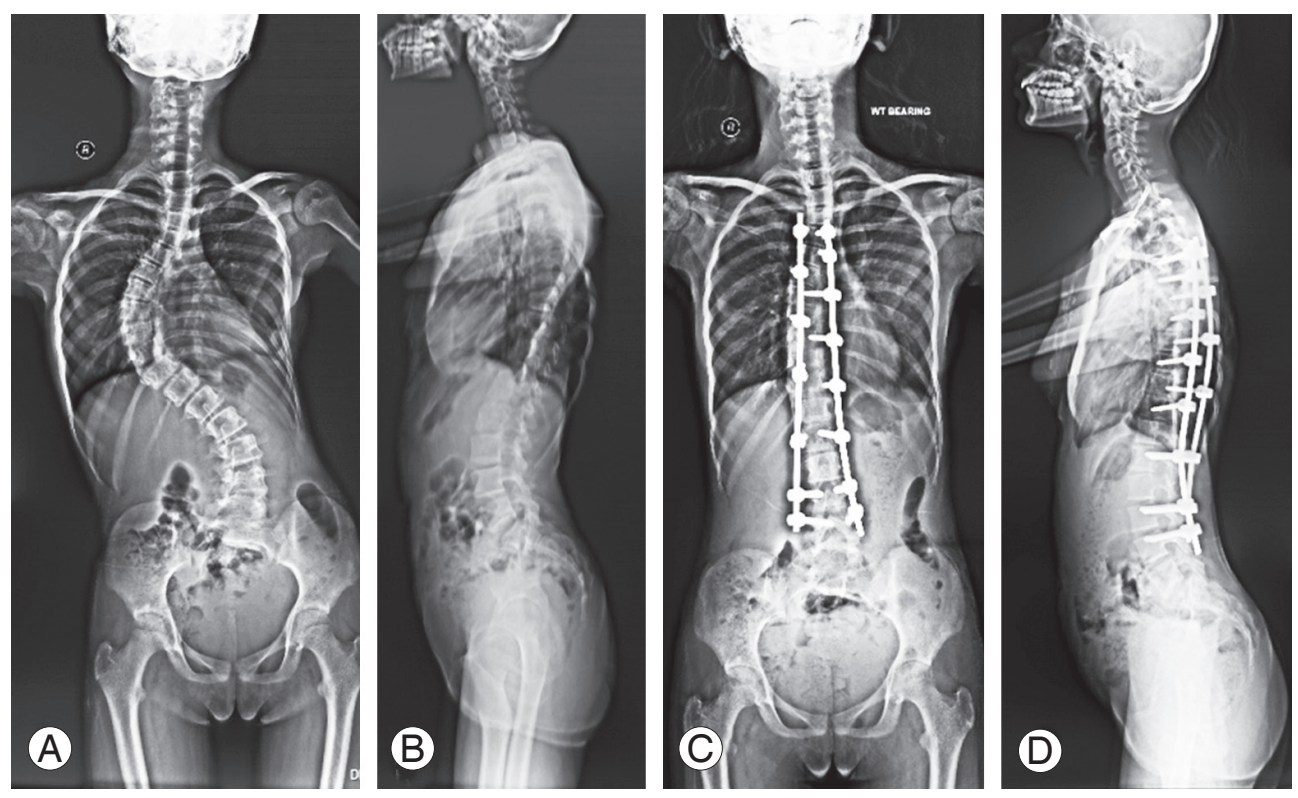

Fig. 2. Preoperative anteroposterior $(A)$ and lateral $(B)$ radiographs showing initial deformation in coronal and sagittal planes. Final follow-up anteroposterior (C) and lateral (D) radiographs showing final correction achieved in coronal and sagittal planes.

rucha et al. [12], in a comparative study on low and HD constructs reported a $66 \%$ correction with LD constructs and found no difference in curve correction between the two groups. In the present study, we achieved $67 \%$ correction in coronal plane with a primary curve flexibility of $41 \%$ and an implant density of $68 \%$. Our results are comparable to studies conducted by Morr et al. [17] and Tannous et al. [18], in which they reported $66.6 \%$ and $66.9 \%$ improvements in the primary curve, respectively. In comparison to Davis and Dunn [7], we achieved better coronal correction attributable to increased implant density in the present study. With regard to CI, a shift of the plumb line toward CSVL ( -7.2 to $-3.4 \mathrm{~mm}$ ) was observed. This indicates an improvement in coronal alignment of the entire spine over the pelvis.

\section{Sagittal parameters}

Restoration of sagittal alignment is essential to achieve a balanced posture where the spine and pelvis are aligned to minimize energy expenditure. Sagittal alignment of the spine can be evaluated via regional (cervical, thoracic, and lumbar), sacro-pelvic, and global sagittal parameters. In normal adolescent populations, Mac-Thiong [19] and Vedantam et al. [20] reported values of $43.0^{\circ} \pm 10.4^{\circ}$ and $38^{\circ} \pm 10^{\circ}$ for TK and $48.4^{\circ} \pm 12.4^{\circ}$ and $64^{\circ} \pm 10^{\circ}$ for LL, re- spectively. A study on sacro-pelvic parameters by MacThiong et al. [19] found mean PI values of $49^{\circ} \pm 11^{\circ}$ and $8^{\circ} \pm 8^{\circ}$ for PT and $41^{\circ} \pm 8^{\circ}$ for SS in normal adolescents.

\section{Regional parameters}

Hilibrand et al. [21] reported straight (lordosis $<5^{\circ}$ ) or kyphotic cervical alignment in 34/39 patients (89\%) and increase in cervical kyphosis postoperatively. In a study conducted by Canavese et al. [22] (32 patients), average preoperative CSA was $4.0^{\circ} \pm 12.3^{\circ}$ (range, $-30^{\circ}$ to $40^{\circ}$ ) and postoperative average CSA was $1.7^{\circ} \pm 11.4^{\circ}$ (range, $-24^{\circ}$ to $\left.30^{\circ}\right)$ lordosis, suggesting decrease in cervical lordosis after surgery. Ilharrehorde et al. [23] reported preoperative cervical kyphosis of 11.2, which improved to cervical lordosis of 7.5 postoperatively. We noted minimal alteration in cervical lordosis after surgery $\left(10.76^{\circ}\right.$ to $\left.10.56^{\circ}\right)$, which was not significant.

TK values $\left(24.46^{\circ}\right)$ were lower than average compared with those in the normal adolescent group, suggesting hypokyphosis in AIS [10]. Davis and Dunn [7] reported a decrease in TK $\left(5.3^{\circ}\right)$ with LD screw constructs, but this loss in kyphosis was lower than that with HD screw constructs. A comparative study on LD and HD screw constructs by Bharucha et al. [12] and Shen et al. [13] showed a decrease in TK by $2.4^{\circ}$ and $2.6^{\circ}$, with LD screw 
constructs, and $5.0^{\circ}$ and $7.4^{\circ}$, with HD screw constructs, respectively. This suggests a greater reduction in TK with HD screw constructs. On the contrary, Wang et al. [14] noted an increase in the average TK with LD screw constructs. We noted a decrease in TK by $1.7^{\circ}(6 \%)$, which is similar to those in studies by Bharucha et al. [12] and Shen et al. [13]. However, in subgroup analysis, the hypokyphosis group showed a $138 \%$ increase $\left(7.0^{\circ}\right.$ to $\left.16.7^{\circ}\right)$ and the hyperkyphosis group showed a $47.2 \%$ decrease $\left(48.7^{\circ}\right.$ to $\left.25.7^{\circ}\right)$ in TK. In the normokyphosis group, there was only a $4 \%$ reduction $\left(24.4^{\circ}\right.$ to $\left.23.3^{\circ}\right)$ suggestive of a slight decrease in TK.

Further, LL was $50.3^{\circ}$, which was lower than that in normal adolescents. This suggests that adolescents affected by scoliosis exhibit hypolordosis in addition to hypokyphosis [10]. Surgery slightly increased the mean value to $51.1^{\circ}$, similar to that reported in a study by Shen et al. [13]. Analysis of the Lenke V subgroup where LL was most severe showed a significant increase in LL of $3.4^{\circ}\left(47.8^{\circ}\right.$ to $\left.51.2^{\circ}\right)$

\section{Sacro-pelvic parameters}

Farshad et al. [24] reported a mean PI of $44^{\circ} \pm 8^{\circ}$, a PT of $34^{\circ} \pm 7^{\circ}$, and an SS of $10^{\circ} \pm 7^{\circ}$ in AIS patients, while Roussouly et al. [25] reported preoperative values of PI, PT, and SS of $52.9^{\circ} \pm 12.4^{\circ}, 10.8^{\circ} \pm 7.7^{\circ}$, and $42.2^{\circ} \pm 8.8^{\circ}$, respectively, and postoperative values of $53.4^{\circ} \pm 12.7^{\circ}, 12.5^{\circ} \pm 7.9^{\circ}$, and $40.9^{\circ} \pm 8.9^{\circ}$, respectively, in AIS patients. We noted mean preoperative PI values of $52^{\circ} \pm 10^{\circ}$, SS values of $37^{\circ} \pm 8^{\circ}$, and PT values of $15^{\circ} \pm 8^{\circ}$. Higher values of PI and PT were noted in our study group, indicating increased retroversion of the pelvis $[23,25]$. Postoperatively, there was an increase in $\mathrm{SS}$ of $3^{\circ}$ with a reciprocal decrease in $\mathrm{PT}$ of $1^{\circ}$, in contrary to the findings of studies by Roussouly et al. [25] and La Maida et al. [26]. Considering changes in sacro-pelvic alignment, AIS corrective surgery using SPSF can help create slight pelvic anteversion, as manifested by a decrease in PT, coupled to a slight increase in SS.

\section{Global sagittal parameters}

A mean negative sagittal imbalance in C7PL (SVA posterior to $\mathrm{C7PL}$ ) of $8.2 \mathrm{~mm}$ was found in the preoperative cohort, shifting anteriorly to $10.6 \mathrm{~mm}$, which is within the normal physiological range (within $20 \mathrm{~mm}$ ). This result was in contradiction to a study by La Maida et al. [26], which showed a posterior shift of the C7PL, but was in agreement with the observation by Wang et al. [14], showing an anterior shift in C7PL. The SSA showed a decrease of $0.7^{\circ}$ (from $127.5^{\circ}$ to $126.8^{\circ}$ ), with restoration of sagittal balance to normal values. Our results are consistent with those of Mac-Thiong et al. [27] evaluating 709 asymptomatic individuals with a mean SSA of $130.4^{\circ} \pm 8.1^{\circ}$ as well as with those of other studies done in AIS patients by Roussouly et al. [25] (129.4 $4^{\circ}$ decreased to $129.3^{\circ}$ postoperatively) and La Maida et al. [26] (131.1 ${ }^{\circ}$ decreased to $129.5^{\circ}$ postoperatively). We observed that although there was an anterior shift in C7PL, global sagittal alignment assessed by SSA suggested that SPSF maintained global spinal balance.

To the best of our knowledge, the present study is the first attempt to analyze the effects of LD screw constructs on overall sagittal parameters in AIS corrective surgery. We found significant correction of the primary curve and improvement in coronal balance. There was restoration of TK in the hypokyphosis and hyperkyphosis groups, while it was maintained in the normokyphosis group. There were specific limitations in this study. First, the retrospective nature of the study design and inclusion of radiographic parameters alone in the analysis were limiting factors. Further, the surgery used 5.5- $\mathrm{mm}$ titanium rods, which are elastic and may be inadequate to maintain the anticipated correction when used for column derotation. The effect of LD screw constructs would be influenced by the nature of the rods used and the reduction maneuvers used for deformity correction.

\section{Conclusions}

Favorable coronal correction with improvement in sacropelvic parameters and global spinal balance was provided by SPSF. In the hypokyphosis and hyperkyphosis groups, TK was significantly corrected with minimal effect in the normokyphosis group. Therefore, SPSF is a viable option to avoid flatback in AIS corrective surgery while still providing good coronal correction.

\section{Conflict of Interest}

No potential conflict of interest relevant to this article was reported. 


\section{Acknowledgments}

The project was funded by Ganga Orthopaedic Research \& Education Foundation, Coimbatore.

\section{References}

1. Dickson RA. The aetiology of spinal deformities. Lancet 1988;1:1151-5.

2. Suk SI, Kim JH, Kim SS, Lim DJ. Pedicle screw instrumentation in adolescent idiopathic scoliosis (AIS). Eur Spine J 2012;21:13-22.

3. Suk SI, Lee CK, Kim WJ, Chung YJ, Park YB. Segmental pedicle screw fixation in the treatment of thoracic idiopathic scoliosis. Spine (Phila Pa 1976) 1995;20:1399-405.

4. Lehman RA Jr, Lenke LG, Keeler KA, et al. Operative treatment of adolescent idiopathic scoliosis with posterior pedicle screw-only constructs: minimum three-year follow-up of one hundred fourteen cases. Spine (Phila Pa 1976) 2008;33:1598-604.

5. Newton PO, Yaszay B, Upasani VV, et al. Preservation of thoracic kyphosis is critical to maintain lumbar lordosis in the surgical treatment of adolescent idiopathic scoliosis. Spine (Phila Pa 1976) 2010;35:136570.

6. Sud A, Tsirikos AI. Current concepts and controversies on adolescent idiopathic scoliosis: part II. Indian J Orthop 2013;47:219-29.

7. Davis JH, Dunn RN. Limited pedicle screw constructs in adolescent idiopathic scoliosis surgery and clinical correlation. SA Orthop J 2013;12:17-21.

8. Kemppainen JW, Morscher MA, Gothard MD, Adamczyk MJ, Ritzman TF. Evaluation of limited screw density pedicle screw constructs in posterior fusions for adolescent idiopathic scoliosis. Spine Deform 2016;4:33-9.

9. Larson AN, Aubin CE, Polly DW Jr, et al. Are more screws better?: a systematic review of anchor density and curve correction in adolescent idiopathic scoliosis. Spine Deform 2013;1:237-47.

10. Mac-Thiong JM, Labelle H, Charlebois M, Huot MP, de Guise JA. Sagittal plane analysis of the spine and pelvis in adolescent idiopathic scoliosis according to the coronal curve type. Spine (Phila Pa 1976) 2003;28:1404-9.

11. Lagrone MO, Bradford DS, Moe JH, Lonstein JE,
Winter RB, Ogilvie JW. Treatment of symptomatic flatback after spinal fusion. J Bone Joint Surg Am 1988;70:569-80.

12. Bharucha NJ, Lonner BS, Auerbach JD, Kean KE, Trobisch PD. Low-density versus high-density thoracic pedicle screw constructs in adolescent idiopathic scoliosis: do more screws lead to a better outcome? Spine J 2013;13:375-81.

13. Shen $M$, Jiang $H$, Luo $M$, et al. Comparison of low density and high density pedicle screw instrumentation in Lenke 1 adolescent idiopathic scoliosis. BMC Musculoskelet Disord 2017;18:336.

14. Wang F, Xu XM, Lu Y, Wei XZ, Zhu XD, Li M. Comparative analysis of interval, skipped, and key-vertebral pedicle screw strategies for correction in patients with Lenke type 1 adolescent idiopathic scoliosis. Medicine (Baltimore) 2016;95:e3021.

15. Kim YJ, Lenke LG, Cho SK, Bridwell KH, Sides B, Blanke K. Comparative analysis of pedicle screw versus hook instrumentation in posterior spinal fusion of adolescent idiopathic scoliosis. Spine (Phila Pa 1976) 2004;29:2040-8.

16. Li M, Shen Y, Fang X, et al. Coronal and sagittal plane correction in patients with Lenke 1 adolescent idiopathic scoliosis: a comparison of consecutive versus interval pedicle screw placement. J Spinal Disord Tech 2009;22:251-6.

17. Morr S, Carrer A, Alvarez-Garcia de Quesada LI, Rodriguez-Olaverri JC. Skipped versus consecutive pedicle screw constructs for correction of Lenke 1 curves. Eur Spine J 2015;24:1473-80.

18. Tannous OO, Banagan KE, Belin EJ, et al. Lowdensity pedicle screw constructs for adolescent idiopathic scoliosis: evaluation of effectiveness and cost. Global Spine J 2016:2192568217735507. https://doi. org/10.1177/2192568217735507.

19. Mac-Thiong JM, Labelle H, Berthonnaud E, Betz RR, Roussouly P. Sagittal spinopelvic balance in normal children and adolescents. Eur Spine J 2007;16:227-34.

20. Vedantam R, Lenke LG, Keeney JA, Bridwell KH. Comparison of standing sagittal spinal alignment in asymptomatic adolescents and adults. Spine (Phila Pa 1976) 1998;23:211-5.

21. Hilibrand AS, Tannenbaum DA, Graziano GP, Loder RT, Hensinger RN. The sagittal alignment of the cervical spine in adolescent idiopathic scoliosis. J Pediatr Orthop 1995;15:627-32. 
22. Canavese F, Turcot K, de Rosa V, de Coulon G, Kaelin A. Cervical spine sagittal alignment variations following posterior spinal fusion and instrumentation for adolescent idiopathic scoliosis. Eur Spine J 2011;20:1141-8.

23. Ilharreborde B, Vidal C, Skalli W, Mazda K. Sagittal alignment of the cervical spine in adolescent idiopathic scoliosis treated by posteromedial translation. Eur Spine J 2013;22:330-7.

24. Farshad M, Catanzaro S, Schmid SL. The spinopelvic geometry in different Lenke curve types of adolescent idiopathic scoliosis. Spine Deform 2016;4:425-31.
25. Roussouly P, Labelle H, Rouissi J, Bodin A. Pre- and post-operative sagittal balance in idiopathic scoliosis: a comparison over the ages of two cohorts of 132 adolescents and 52 adults. Eur Spine J 2013;22 Suppl 2:S203-15.

26. La Maida GA, Zottarelli L, Mineo GV, Misaggi B. Sagittal balance in adolescent idiopathic scoliosis: radiographic study of spino-pelvic compensation after surgery. Eur Spine J 2013;22 Suppl 6:S859-67.

27. Mac-Thiong JM, Roussouly P, Berthonnaud E, Guigui P. Sagittal parameters of global spinal balance: normative values from a prospective cohort of seven hundred nine Caucasian asymptomatic adults. Spine (Phila Pa 1976) 2010;35:E1193-8. 